\title{
Divergence of Casimir stress in inhomogeneous media
}

\author{
William M. R. Simpson, ${ }^{1,2}$ S. A. R. Horsley, ${ }^{3}$ and U. Leonhardt ${ }^{2}$ \\ ${ }^{1}$ School of Physics and Astronomy, University of St Andrews, North Haugh, St Andrews, KY16 9SS, United Kingdom \\ ${ }^{2}$ Faculty of Physics, Weizmann Institute of Science, 234 Herzl Street, Rehovot 76100, Israel \\ ${ }^{3}$ Electromagnetic and Acoustic Materials Group, Department of Physics and Astronomy, University of Exeter, Stocker Road, Exeter, \\ EX4 4QL, United Kingdom
}

(Received 18 January 2013; published 5 April 2013)

\begin{abstract}
We examine the local behavior of the regularized stress tensor commonly used in calculations of the Casimir force for a dielectric medium inhomogeneous in one direction. It is shown that the usual expression for the stress tensor is not finite anywhere within the medium, whatever the temporal dispersion or index profile, and that this divergence is unlikely to be removed through a simple modification to the regularization procedure. Our analytic argument is illustrated numerically for a medium approximated as a series of homogeneous strips, as the width of these strips is taken to zero. The findings hold for all magnetodielectric media.
\end{abstract}

DOI: 10.1103/PhysRevA.87.043806

PACS number(s): 42.50.Lc, 31.30.jh

\section{INTRODUCTION}

In 1948 Casimir offered an argument for the existence of an attractive force between two parallel uncharged mirrors that exists even at zero temperature [1] $-a$ force arising from the ground-state properties of the electromagnetic field. Yet, calculation of the Casimir force requires a regularization procedure. The quantization of the field results in an infinite contribution to the ground-state energy, a portion of which must be removed before a finite, physical force can be extracted.

A more realistic physical description should include dispersion and dissipation, and Lifshitz theory offers this apparatus [2], one that has been applied to experiments with success [3,4] (although some doubts over Lifshitz theory remain [5], due, e.g., to the strange results it produces for media with a finite de conductivity). The formalism is written in terms of the electromagnetic Green's function, which describes the field produced by sources of current within the system. A stress tensor is written in terms of this Green's function from which a force can be derived. The physical picture behind Lifshitz theory is this: the ground state of the coupled system of electromagnetic field and dielectric is one with nonzero current density within the media [6,7], consistent with the fluctuation dissipation theorem [8]. The Casimir-Lifshitz force arises from the interaction of these currents.

The stress tensor, however, at least contains the same divergent contribution that appeared in Casimir's original work and must also be regularized. Typically this is achieved through subtracting from the total Green's function an auxiliary Green function associated with an infinite homogeneous medium [7,9-12]. One can then compute a finite stress tensor for the system that depends on the dielectric functions of the material at imaginary frequencies (quantities obtained from the dielectric properties for real frequencies by Hilbert transformation). Only then can the force be derived. Both Casimir's and Lifshitz' regularization procedures give identical results in the limiting case of a cavity sandwiched between perfectly reflecting mirrors [11].

In more recent work, it has been found that the aforementioned method of regularization fails to yield finite results when applied to an inhomogeneous dielectric, when the optical properties vary continuously in space [10]. An alternative form for the regularizer has been proposed [10,11], but this does not succeed (see Appendix A). One resolution to this apparent problem could be that the stress tensor is not meaningful within a medium, being irrelevant to the computation of forces in vacuum. However, not only is this point of view fundamentally dissatisfying, but the experimental situation of interest is often that of media embedded within a fluid [3], where the Casimir force must be computed using the stress tensor within the fluid. We might ask for the value of the force in the case when we have an inhomogeneous fluid, and at present it seems that Lifshitz theory cannot provide an answer.

This said, two calculations of Casimir forces in inhomogeneous media have recently been presented $[13,14]$. In [13] it seems the authors do not yet extract numerical results from their formalism. An explicit construction of a regularization procedure that goes beyond that used by Lifshitz has apparently not yet been given, and we suspect that it is not straightforward to do so. Meanwhile, in [14], the concern is with the Casimir force between two mirrors with an inhomogeneous medium sandwiched between them. Here the force is calculated, not via Lifshitz theory but from mode summation, obtaining finite results using techniques similar to $\zeta$-function regularization. A function $f(s)$ with a free parameter $s$ is inserted into the summand to force convergence, and then the resultant pressure on the mirrors is Laurent expanded around $s=0$, where $f(0)=1$, throwing away negative powers of $s$. The result is clearly finite, but it is not clear that the diverging terms are independent of the position of the mirrors, which they must be if they are not to contribute to the force. Moreover, we believe that consistently including the effects of dispersion and dissipation will inevitably lead one to a theory written in terms of Green's functions, with the inherent regularization problem that we highlight.

Here we extend the analysis of [10] in an attempt to find the origin of this problem, and to examine the prospects for obtaining the electromagnetic stress within an inhomogeneous medium. According to our treatment, it seems unlikely that any simple modification of the regularization procedure will be successful. We illustrate the development of the divergence in the passage from a piecewise definition of a medium to a 
continuous one, finding that-without the input of additional physics - it seems necessary to make a somewhat arbitrary correction to the Green's function in order to make the stress finite.

\section{THE REGULARIZED STRESS IN THE CONTINUUM LIMIT}

The usual expression for the stress tensor, when applied to a medium that is piecewise defined along a single axis, is known to be finite. This is as long as we do not ask for all of the components of the stress tensor as we approach the boundaries of the homogeneous regions [15]. To be explicit, for a region of width $a$ where $\epsilon$ and $\mu$ are homogeneous, the value of the regularized stress tensor at a point $x$ can be written in terms of the reflection coefficients (as opposed to the Green's functions) associated with sending $q$-polarized $(q=s, p)$ plane waves to the right $\left(r_{q R}\right)$ and to the left $\left(r_{q L}\right)$ of this point $[11,16,17]$,

$$
\sigma_{x x}(x)=2 \hbar c \sum_{q=s, p} \int_{0}^{\infty} \frac{d \kappa}{2 \pi} \int_{\mathbb{R}^{2}} \frac{d^{2} \boldsymbol{k}_{\|}}{(2 \pi)^{2}} w \frac{r_{q L} r_{q R} e^{-2 a w}}{1-r_{q L} r_{q R} e^{-2 a w}},
$$

where $w=\left(n^{2} \kappa^{2}+k_{\|}^{2}\right)^{1 / 2}, k_{\|}=\left|\boldsymbol{k}_{\|}\right|$, and $n$ is the value of the refractive index in the homogeneous region surrounding $x$. The reflection coefficients are functions of the imaginary frequency $\omega=i c \kappa$, the (real) in-plane wave vector $\boldsymbol{k}_{\|}$, and the material parameters of the media to the right and to the left of the homogeneous region. The advantage of writing the stress tensor in this form is that the regularization procedure of Lifshitz theory is automatically implemented [11]. The contributions to the stress arise entirely from inhomogeneities in the system. Here we investigate the behavior of (1) in the limit as the piecewise definition of the medium becomes a continuous function $(a \rightarrow 0)$.

\section{GENERAL ARGUMENT FOR MEDIA INHOMOGENEOUS IN ONE DIRECTION}

We initially approximate the inhomogeneous medium as a series of $N$ homogeneous strips of width $a$ (see Fig. 1), only taking the limit of $N \rightarrow \infty$ and $a \rightarrow 0$ in the final step of the calculation. The transfer matrix technique can be used for such

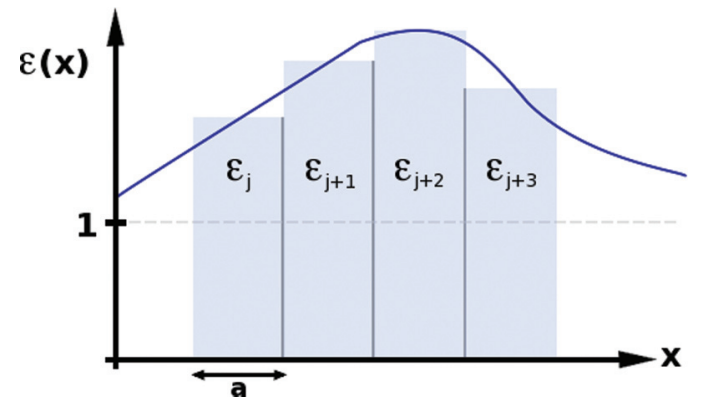

FIG. 1. (Color online) The medium is assumed inhomogeneous along $x$ and is divided into $N$ homogeneous slices of width $a$. The local value of the regularized stress tensor (1) is then investigated within the medium in the limit as $a \rightarrow 0$. For the purposes of illustration only the permittivity $\epsilon$ is shown here. Our analysis holds for both inhomogeneous permittivity and permeability. an analysis of the field [17-20], the field in strip $j+1$ being related to that in $j$ by

$$
\boldsymbol{E}_{q}(j+1)=\boldsymbol{t}_{q}(j+1) \cdot \boldsymbol{E}_{q}(j) .
$$

The index $q$ labels the polarization as in (1), and $\boldsymbol{t}_{q}(j+1)$ is the transfer matrix relating the field on the far right of slice $j$ to that on the far right of slice $j+1$. In (2) the electric field amplitude $\boldsymbol{E}_{q}$ is written as a two-element vector containing the right- $(+)$ and left- $(-)$ going parts,

$$
\boldsymbol{E}_{q}(j)=\left(\begin{array}{c}
E_{q}^{(+)}(j) \\
E_{q}^{(-)}(j)
\end{array}\right) \text {. }
$$

We number the transfer matrices in (2) from 1 to $N+1$, with $\epsilon_{0}$ and $\epsilon_{N+1}$ equal to the vacuum permittivity, and $\mu_{0}$ and $\mu_{N+1}$ the vacuum permeability. In each of these slices $\boldsymbol{t}_{q}$ is given by the usual expression for the transfer matrix in piecewise homogeneous media (e.g., [18,20]). For the imaginary frequencies, $\omega / c=i \kappa$, encountered within (1) the $x$-directed wave vector in the $j$ th slice is also imaginary, $k_{j}=i w_{j}$, where $w_{j}=\left(n_{j}^{2} \kappa^{2}+k_{\|}^{2}\right)^{1 / 2}$.

We aim to apply this formalism to show that the stress tensor (1), though regularized, fails to be finite when the properties of the medium are represented by continuous functions of position. The divergence of (1) is anticipated to come from the integral over $k_{\|}=\left|\boldsymbol{k}_{\|}\right|$. Physically, considering the allowed modes on the real frequency axis, we can picture this divergent contribution arising due to waves of high $k_{\|}$undergoing reflections from the inhomogeneity of the medium. As $k_{\|}$ is increased within the integrand, the contribution of these waves to the local value of the stress tensor fails to diminish sufficiently rapidly, when presumably in reality they should not be supported by the medium at all.

To understand the reason for our suspicion, before proceeding with a more lengthy argument below, consider (1) in a cavity of width $a$ (see Fig. 1). This quantity is finite when we integrate over $k_{\|}$due to the exponential decay associated with propagation across the cavity, which is increasingly rapid as $k_{\|}$increases. Indeed, once $k_{\|}$becomes sufficiently large, then the field cannot reach the boundaries of the cavity at all and the reflection coefficients correspondingly tend to zero. However, upon shrinking $a$, this convergence becomes slower, a higher value of $k_{\|}$being required before the field fails to make a round trip across the cavity. Given that a continuous medium can be understood as the limit where $a \rightarrow 0$, and the refractive index contrast between the cavity and the walls becomes infinitesimal, we should ask whether the reflection coefficients vanish fast enough as $a \rightarrow 0$ in order for the stress (1) to be finite. It seems that they do not: changing variables in (1) to $\zeta=a w$, and $\xi=a k_{\|}$, we find the whole integral multiplied by $a^{-3}$. Meanwhile, in this limit the reflection coefficients would in general have contributions linear in $a$ (this being the order of magnitude of the contrast in $\epsilon$ and $\mu$ between the cavity and the walls), which would still leave a term proportional to $a^{-1}$ within the stress tensor-a term which diverges in the continuum limit.

We shall now attempt to make these rough arguments more precise. For the purpose of identifying this anticipated divergence, we restrict our attention to the regime of the integrand in (1) where the in-plane wave vector is large 
in comparison to the "refractive index" multiplied by the "frequency", $n_{j} \kappa / k_{\|} \ll 1$. The quantity $w_{j}$ then becomes constant throughout the medium $w_{j} \sim k_{\|}$and the transfer matrices simplify to

$$
\begin{aligned}
& \boldsymbol{t}_{1}(j+1)=\boldsymbol{\alpha}+\frac{\Delta \mu_{j}}{2 \mu_{j}} \boldsymbol{\beta}, \\
& \boldsymbol{t}_{2}(j+1)=\frac{n_{j+1} \epsilon_{j}}{n_{j} \epsilon_{j+1}}\left(\boldsymbol{\alpha}+\frac{\Delta \epsilon_{j}}{2 \epsilon_{j}} \boldsymbol{\beta}\right),
\end{aligned}
$$

where $\Delta \mu_{j}=\mu_{j+1}-\mu_{j}, \Delta \epsilon_{j}=\epsilon_{j+1}-\epsilon_{j}$, and

$$
\boldsymbol{\alpha}=\left(\begin{array}{cc}
e^{-k_{\|} a} & 0 \\
0 & e^{k_{\|} a}
\end{array}\right), \quad \boldsymbol{\beta}=\left(\begin{array}{cc}
e^{-k_{\|} a} & -e^{-k_{\|} a} \\
-e^{k_{\|} a} & e^{k_{\|} a}
\end{array}\right) .
$$

We now examine the field at a fixed point in the medium $x_{l}$, that is, within the $l$ th slice. To calculate the value of (1), at this point we require expressions for both $r_{R}$ and $r_{L}$. These can be calculated in terms of the transfer matrices $\boldsymbol{T}_{R}$ and $\boldsymbol{T}_{L}$ that are respectively associated with propagation through the medium to the right and to the left of $x_{l}$. The expressions for these quantities are

$$
\boldsymbol{T}_{q L}=\prod_{j=1}^{l} \boldsymbol{t}_{q}(j), \quad \boldsymbol{T}_{q R}=\prod_{j=l+1}^{N+1} \boldsymbol{t}_{q}(j) .
$$

It is not possible to analytically evaluate (6) unless we make a further approximation. The approximation we make is equivalent to the Born approximation in quantum mechanics, where we assume that scattering is weak [19,21]: in electromagnetism this means that the properties of the medium must change slowly as a function of position. Products of the transfer matrices can then be truncated to first order in $\Delta \epsilon$ and $\Delta \mu$ [18]. Such an approximation is quite well suited to our situation, for it is the case where the value of the stress ought to be minimal. Inserting (4) and truncating the products (6) to first order, we have, for example,

$$
\boldsymbol{T}_{1 L} \sim\left(\begin{array}{cc}
{\left[1+\sum_{j} \frac{\Delta \mu_{j}}{2 \mu_{j}}\right] e^{-k_{\|} l a}} & -\sum_{j} \frac{\Delta \mu_{j}}{2 \mu_{j}} e^{-k_{\|}(l-2 j) a} \\
-\sum_{j} \frac{\Delta \mu_{j}}{2 \mu_{j}} e^{k_{\|}(l-2 j) a} & {\left[1+\sum_{j} \frac{\Delta \mu_{j}}{2 \mu_{j}}\right] e^{k_{\|} l a}}
\end{array}\right), \quad \boldsymbol{T}_{1 R} \sim\left(\begin{array}{cc}
{\left[1+\sum_{j} \frac{\Delta \mu_{j}}{2 \mu_{j}}\right] e^{-k_{\|}(N-l+1) a}} & -\sum_{j} \frac{\Delta \mu_{j}}{2 \mu_{j}} e^{-k_{\|}(N+l+1-2 j) a} \\
-\sum_{j} \frac{\Delta \mu_{j}}{2 \mu_{j}} e^{k_{\|}(N+l+1-2 j) a} & {\left[1+\sum_{j} \frac{\Delta \mu_{j}}{2 \mu_{j}}\right] e^{k_{\|}(N-l+1) a}}
\end{array}\right)
$$

where the summation over $j$ runs from 0 to $l-1$ in $\boldsymbol{T}_{1 L}$ and from $l$ to $N$ in $\boldsymbol{T}_{1 R}$. As can be seen from (4), the form of $\boldsymbol{T}_{2 L}$ and $\boldsymbol{T}_{2 R}$ only differs in replacing permeability with permittivity and multiplying by a prefactor, which does not affect the reflection coefficients.

In the continuum limit, the summations within (7) become integrals over quantities involving the logarithmic derivative of $\mu$ with respect to $x$. Had we not truncated (6) to first order in $\Delta \mu_{j}$, we would also have a series of additional terms that in the continuum limit become products of integrals over higher powers of the first derivative of $\mu$. For example, the secondorder correction to $\boldsymbol{T}_{1 L}$ is given in Appendix B.

Applying the usual expressions for the reflection coefficients in terms of ratios of the elements of the transfer matrix [22], we calculate $r_{q L}$ and $r_{q R}$ from (7) and the equivalent quantities for the second polarization. We find for the first polarization,

$$
\begin{aligned}
& r_{1 L}(x)=-\frac{\sum_{j=0}^{l-1} \frac{\Delta \mu_{j}}{\mu_{j}} e^{-2 k_{\|}(l-j-1 / 2) a}}{\left(2+\sum_{j=0}^{l-1} \frac{\Delta \mu_{j}}{\mu_{j}}\right)}, \\
& r_{1 R}(x)=\frac{\sum_{j=l}^{N} \frac{\Delta \mu_{j}}{\mu_{j}} e^{-2 k_{\|}(j-l+1 / 2) a}}{\left(2+\sum_{j=l}^{N} \frac{\Delta \mu_{j}}{\mu_{j}}\right)} .
\end{aligned}
$$

The reflection coefficients for the second polarization are of an identical form but with permeability replaced by permittivity. The additional factors of $a / 2$ within the exponentials have been introduced such that the point $x_{l}$ is at the center of the $l$ th slice.

The integrand of (1) is evaluated at a fixed $\kappa$, and a semiinfinite part of the integral over $k_{\|}$is taken, $[K, \infty)$, where, on the basis of our earlier discussion, we expect this expression to diverge and where it is assumed that the reflection coefficients can be taken as (8) to within a reasonable approximation:

$$
\begin{aligned}
I & =\sum_{q} \int_{0}^{2 \pi} \frac{d \theta}{2 \pi} \int_{K}^{\infty} \frac{k_{\|}^{2} d k_{\|}}{2 \pi} \frac{r_{q L} r_{q R} e^{-2 k_{\|} a}}{1-r_{q L} r_{q R} e^{-2 k_{\|} a}} \\
& =\frac{1}{2 \pi} \sum_{n=0}^{\infty} \sum_{q} I_{q n} .
\end{aligned}
$$

In the final step we have expanded the denominator in a series of ascending powers of the reflection coefficients. We assume that $r_{q L} r_{q R} e^{-2 k_{\|} a}<1$ so that this sum converges for all $a$ and all $k_{\|}$. It is then possible to interchange the order of integration and summation, and we introduce the quantities

$$
I_{q n}=\int_{K}^{\infty} k_{\|}^{2} d k_{\|}\left(r_{q L} r_{q R}\right)^{n+1} e^{-2(n+1) k_{\|} a} .
$$

The expressions given in (8) are now inserted into (10), and then the integral over $k_{\|}$in all these terms can be evaluated, yielding, for instance,

$$
I_{10}=-\frac{\sum_{j=0}^{l-1} \sum_{k=l}^{N} \frac{\Delta \mu_{j} \Delta \mu_{k}}{\mu_{j} \mu_{k}}\left[\frac{K^{2}}{2(k-j) a}+\frac{2 K}{4(k-j)^{2} a^{2}}+\frac{2}{8(k-j)^{3} a^{3}}\right] e^{-2 K(k-j) a}}{\left(2+\sum_{j=0}^{l-1} \frac{\Delta \mu_{j}}{\mu_{j}}\right)\left(2+\sum_{j=l}^{N} \frac{\Delta \mu_{j}}{\mu_{j}}\right)},
$$


with the further terms containing higher powers of the $\Delta \mu_{i}$. The expression given in (11) can be increased to an arbitrarily large value through decreasing the width of the slicing due to the terms within the summand around $k-j=1$. To see this, consider the continuum limit $\left(x_{l} \rightarrow x\right)$. In the limit, (11) becomes

$$
I_{10} \rightarrow-\frac{\int_{0}^{x} d x_{1} \int_{x}^{L} d x_{2} \frac{d \ln \left[\mu\left(x_{1}\right)\right]}{d x_{1}} \frac{d \ln \left[\mu\left(x_{2}\right)\right]}{d x_{2}}\left[\frac{K^{2}}{2\left(x_{2}-x_{1}\right)}+\frac{2 K}{4\left(x_{2}-x_{1}\right)^{2}}+\frac{2}{8\left(x_{2}-x_{1}\right)^{3}}\right] e^{-2 K\left(x_{2}-x_{1}\right)}}{\{2+\ln [\mu(x)]\}\{2-\ln [\mu(x)]\}} .
$$

This expression clearly diverges. It therefore seems that there is no finite continuum limit of the regularized stress tensor (1). It is important to emphasize that including the additional terms in the series (9), as well as corrections such as (B1), does not affect this result. These contributions diverge in a similar manner but represent higher powers of the derivatives of $\epsilon$ and $\mu$-terms that vary quite independently as the spatial dependence of $\epsilon$ and $\mu$ is changed, and therefore cannot be expected to cancel in general. As the remainder of the integral over $\boldsymbol{k}_{\|}$is finite, we conclude that the whole integral diverges as $a \rightarrow 0$. Consequently (1) diverges everywhere within an inhomogeneous medium described by $\epsilon$ and $\mu$ that are continuous functions of position. This is independent of how these quantities depend on imaginary frequency.

We might wonder how finite results ought to be extracted from this formalism. The advantage of the usual regularization procedure is that it removes an infinite quantity that does not depend on the inhomogeneity of the medium, and such a quantity cannot be relevant to the force. Conversely, here we have a divergent contribution that is due to the inhomogeneity of the medium, and it is not clear what should be subtracted to obtain the (finite) physical value of the stress tensor. The divergence originates within the fact that the reflection coefficients (8) do not go to zero fast enough as $k_{\|} \rightarrow \infty$ in the limit where $a \rightarrow 0$. Therefore we could terminate the integral over $k_{\|}$at some finite cutoff. However, the value of this cutoff would be a matter of choice. Alternatively, before the continuum limit is taken in (11), we might just remove some small region of the sum around the point where $k-j=1$, although the size of this region would also be arbitrary.

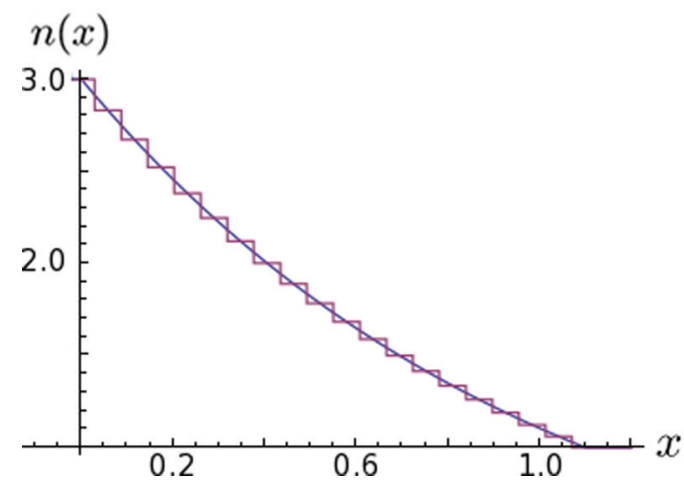

FIG. 2. (Color online) The continuous refractive index profile of the system and a piecewise approximation using 20 homogeneous slices.
This problem is reminiscent of that found in the theory of spontaneous emission within an absorbing dielectric, where an additional physical parameter-equivalent to removing a portion of the dielectric in the immediate vicinity of the atom-must be introduced in order to obtain a finite emission rate $[23,24]$.

\section{NUMERICAL ILLUSTRATION}

The divergence demonstrated analytically above was first spotted numerically when attempting to compute a stress profile for a system similar to the one in [10], and the results of these numerical computations serve to illustrate the argument. For the sake of simplicity we consider an impedance-matched system $\epsilon=\mu=n$ with the refractive index profile

$$
n(x)= \begin{cases}3 & x \leqslant 0 \\ 3 \mathrm{e}^{-x} & 0<x<\ln (3) \\ 1 & x \geqslant \ln (3) .\end{cases}
$$

The system contains an inhomogeneous region between $x=0$ and $x=\ln (3)$. In order to investigate the properties of this system using the transfer matrix technique described earlier [but dispensing with the approximations (7)], we divide the inhomogeneous region into $N$ homogeneous pieces (see Fig. 2) and determine the left and right reflection coefficients within each piece. It is then possible to calculate the local value of the regularized stress. The formula for the stress (1) can be rewritten more simply in this case [11], noting that the coefficients depend only on the magnitude of the wave-vector

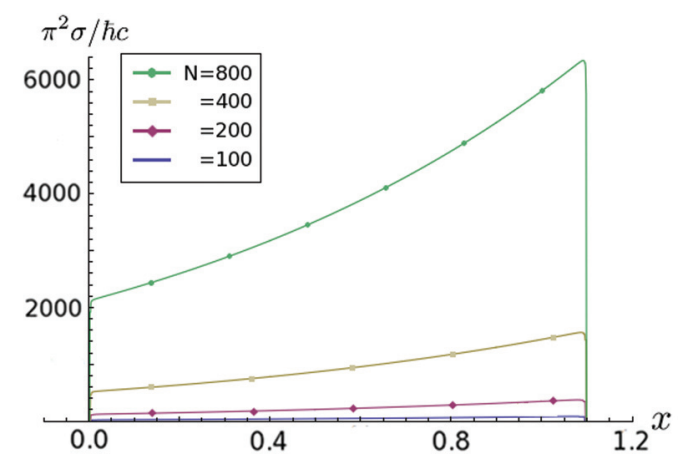

FIG. 3. (Color online) The medium, inhomogeneous between $x=0$ and $x=\ln (3)$, is divided into $100,200,400$, and 800 homogeneous slices. The local absolute value of the regularized stress tensor (13) - normalized in units of $\hbar c / \pi^{2}$-is plotted for each case at a given position $x$. The stress increases as the number of divisions increases. 


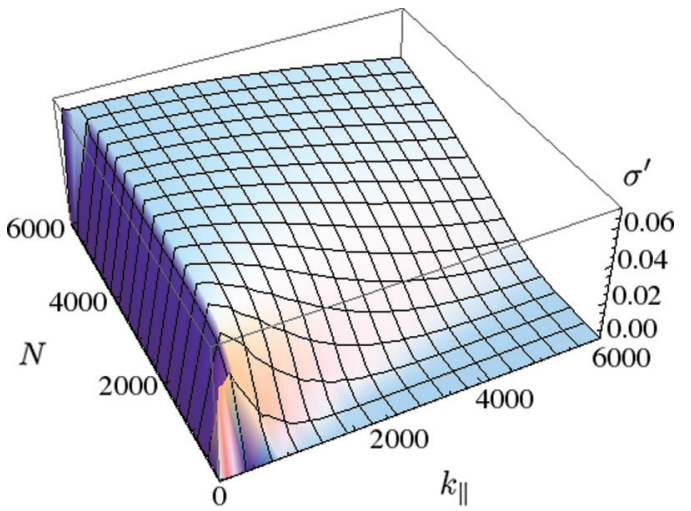

FIG. 4. (Color online) The integrand of the stress (13) $\sigma^{\prime}$ (normalized in the same units as Fig. 3) is plotted for $\kappa=1$ at the center of the system, with $k_{\|}$varying from $k_{\|}=1$ to $k_{\|}=6000$ (horizontal axis), and $N$ ranging from $t=10$ to $t=6000$ (depth axis). As the number of slices $N$ is increased, the integrand falls off less rapidly with $k_{\|}$, and thus the integral of the stress converges less rapidly.

components and not on the angle between them [25]:

$$
\sigma=\frac{\hbar c}{\pi^{2}} \int_{0}^{\infty} \int_{0}^{\infty} k_{\|} w \frac{r_{L} r_{R} e^{-2 a w}}{1-r_{L} r_{R} e^{-2 a w}} d k_{\|} d \kappa .
$$

As $N$ becomes large (i.e., as the cavity width $a$ becomes small), the approximation becomes increasingly accurate. Prima facie, there should be little to distinguish the physics of the case $N=400$ from the case $N=800$, as both approximations of the continuum case are now very smooth. Nevertheless, as Fig. 3 shows, the stress (though regularized) increases markedly and it continues to grow as more slices are added. Why is this happening? Plots of the integrand of the stress (13), where the wave number $k_{\|}$and the number of slices $N$ are allowed to vary, show that the integral falls off less and less rapidly with $k_{\|}$as $N$ is increased (Fig. 4).

\section{CONCLUSIONS}

From our investigation, it is clear that a calculation of (1) for a piecewise definition of an inhomogeneous medium does not represent an approximation to the continuous case. We have found that the expression for the stress tensor commonly employed in calculations of the Casimir force is not finite anywhere within an inhomogeneous medium. Our result is consistent with the findings of [10] and illustrates the generality of the problem of specifying the local value of the electromagnetic stress tensor at $T=0 \mathrm{~K}$ when the material parameters vary continuously over space. Moreover, we identify a divergence of the local value of the stress tensor that cannot be removed by the procedure of regularization usually advocated; it arises specifically due to the unphysical contribution of high wave numbers in the continuum limit. This problem does not seem to be widely appreciated in the literature. In [17] and in [26], reflection coefficients were similarly employed to determine the Casimir force in systems with increasingly refined inhomogeneous features, but the limits of the applicability of this technique were not commented on.

One possible explanation for the divergence we identify is that the Casimir force does not in fact depend on such smallscale inhomogeneities as a continuously varying medium introduces, and an additional physical parameter, perhaps equivalent to removing a portion of the dielectric around the measuring point, could be required (cf. [23,24]). If so, it seems unlikely that a generally finite and physically meaningful result could be obtained through a simple modification to the existing regularization procedure; such an additional parameter would presumably depend upon the microphysical properties of the material. Alternatively, perhaps the sensitivity of the Casimir force to microscopic discontinuities simply cannot be described by classical wave propagation. A modification to the regularization in that case might still be possible, although it is difficult to see what this may involve. Either way, there is evidently additional physics to take into account on this scale to address the problem of the Casimir force in inhomogeneous media. The correct solution, however, is not something that can be deduced directly from the piecewise approximation.

\section{ACKNOWLEDGMENTS}

S.A.R.H. and W.M.R.S. acknowledge illuminating conversations with T. G. Philbin (Exeter), Jim Fowler (Ohio), and Steven Johnson (MIT), and also financial support from the EPSRC and from SUPA.

\section{APPENDIX A: FAILURE OF MODIFIED REGULARIZATION}

In [10] a modification of the standard regularizer is considered. This also appears in [11], where a general recipe for calculating finite Casimir-Lifshitz forces is proffered. One of the drawbacks of Lifshitz theory is the difficulty of obtaining an analytic Green's function for the system under consideration (in this case, an inhomogeneous system), which makes this procedure difficult to apply and such proposals difficult to test. We will not recapitulate the details of the procedure here (the reader is referred instead to [11]). In our test case, we applied it to an impedance-matched system with an inhomogeneous refractive index profile of

$$
\epsilon(r)=\mu(r)=n(r)=\frac{2}{1+r^{2}},
$$

a system investigated by two of the authors of [10] themselves in a different context, for which they determined the exact Green's function [10], and a system where the Casimir-Lifshitz force is known independently of the regularization [27]. The Green's function of the system is

$$
G\left(r, r^{\prime}\right)=\frac{\nabla \times n\left(r_{m}\right) \nabla \otimes \nabla^{\prime} D\left(r_{m}\right) \times \overleftarrow{\nabla^{\prime}}}{n(r) n\left(r^{\prime}\right) k^{2}},
$$

where $D$ is the scalar function,

$$
D_{0}\left(r_{m}\right)=\frac{1}{8 \pi}\left(r_{m}+\frac{1}{r_{m}}\right) \exp \left(2 i k \arctan r_{m}\right),
$$


the quantity $r_{m}$ is the Mobius length, defined by

$$
r_{m}=\frac{\left|r-r^{\prime}\right|}{\sqrt{1+2 r \cdot r^{\prime}+r^{2} r^{\prime 2}}},
$$

and $r$ and $r^{\prime}$ are the measuring and source points, respectively. Regularization is effected by subtracting from the Green's function of the electric field a regularizing Green's function,

$$
G_{\mathrm{phys}}=G-G_{0} \text {. }
$$

Following the recipe offered in [11] for constructing the regularizer, we obtain

$$
G_{0}\left(r, r^{\prime}\right)=-\left(\frac{\nabla \otimes \nabla^{\prime}}{\sqrt{\varepsilon \varepsilon^{\prime}} \kappa^{2}}+\sqrt{\mu \mu^{\prime}} \mathbb{1}_{3}\right) g_{0},
$$

where $g_{0}$ is the scalar Green's function,

$$
g_{0}\left(r, r^{\prime}\right)=-\frac{1}{4 \pi r} \exp \left(-2 \kappa \arctan r_{m}\right) .
$$

A Casimir-Lifshitz force can be obtained, according to the recipe, by computing the divergence of the stress tensor in the limit of the spectator point approaching the source point. Upon computing the stress for $G_{\text {phys }}$, however, and taking the divergence, we find a Casimir force that diverges everywhere in the system as $1 / r^{2}$. We conclude that this procedure fails to provide a general method of regularization that removes all the divergences in the stress tensor.

\section{APPENDIX B: HIGHER-ORDER CORRECTIONS TO THE TRANSFER MATRICES}

The second-order correction to the expression $\boldsymbol{T}_{1 L}$ in (6) is

$$
\begin{aligned}
& \sum_{j=0}^{l-2} \sum_{k=0}^{l-2}\left(\frac{\Delta \mu_{j}}{2 \mu_{j}}\right)\left(\frac{\Delta \mu_{j+k+1}}{2 \mu_{j+k+1}}\right) \boldsymbol{\alpha}^{(l-2)-j-k} \boldsymbol{\beta} \boldsymbol{\alpha}^{k} \boldsymbol{\beta} \boldsymbol{\alpha}^{j} \\
& \quad=\sum_{j=0}^{l-2} \sum_{k=0}^{l-2}\left(\frac{\Delta \mu_{j}}{2 \mu_{j}}\right)\left(\frac{\Delta \mu_{j+k+1}}{2 \mu_{j+k+1}}\right)\left(\begin{array}{cc}
e^{-k_{\|} l a}\left(1+e^{2 k_{\|}(k+1) a}\right) & -e^{-k_{\|}(l-2 j) a}\left(1+e^{2 k_{\|}(k+1) a}\right) \\
-e^{k_{\|}(l-2 j) a}\left(1+e^{-2 k_{\|}(k+1) a}\right) & e^{k_{\|} l a}\left(1+e^{-2 k_{\|}(k+1) a}\right)
\end{array}\right),
\end{aligned}
$$

each element of which in the continuum limit becomes a double integral over a quantity involving the second power of the logarithmic derivative of $\mu$.

[1] H. B. G. Casimir, Koninkl. Ned. Akad. Wetenschap. 51, 793 (1948).

[2] I. E. Dzyaloshinskii, E. M. Lifshitz, and L. P. Pitaevskii, Adv. Phys. 10, 165 (1961).

[3] J. N. Munday, F. Capasso, and V. A. Parsegian, Nature (London) 457, 170 (2009).

[4] A. W. Rodriguez, F. Capasso, and S. G. Johnson, Nat. Photonics 5, 211 (2011).

[5] G. L. Klimchitskaya, U. Mohideen, and V. M. Mostepanenko, Rev. Mod. Phys. 81, 1827 (2009).

[6] T. G. Philbin, New J. Phys. 12, 123008 (2010).

[7] T. G. Philbin, New. J. Phys. 13, 063026 (2011)

[8] L. D. Landau and E. M. Lifshitz, Statistical Physics (Part 1) (Butterworth-Heinemann, Oxford, UK, 2005).

[9] E. M. Lifshitz and L. P. Pitaevskii, Statistical Physics (Part 2) (Butterworth-Heinemann, Oxford, UK, 2003).

[10] T. G. Philbin, C. Xiong, and U. Leonhardt, Ann. Phys. 325, 579 (2009).

[11] U. Leonhardt, Quantum Optics (Cambridge University Press, Cambridge, UK, 2010).

[12] L. P. Pitaevskii, Casimir Physics (Springer, Berlin, 2011).

[13] F. S. S. Rosa, D. A. R. Dalvit, and P. W. Milonni, Phys. Rev. A 84, 053813 (2011).

[14] S. I. Goto, A. C. Hale, R. W. Tucker, and T. J. Walton, Phys. Rev. A 85, 034103 (2012).

[15] T. G. Philbin (private communication).
[16] E. M. Lifshitz, Zh. Eksp. Teor. Fiz. 29, 94 (1955).

[17] C. Genet, A. Lambrecht, and S. Reynaud, Phys. Rev. A 67, 043811 (2003).

[18] M. Born and E. Wolf, Principles of Optics (Cambridge University Press, Cambridge, UK, 1999).

[19] I. A. Shelykh and V. K. Ivanov, Int. J. Theor. Phys. 43, 477 (2004)

[20] M. Artoni, G. C. La Rocca, and F. Bassani, Phys. Rev. E 72, 046604 (2005).

[21] L. D. Landau and E. M. Lifshitz, Quantum Mechanics (Butterworth-Heinemann, Oxford, 2003).

[22] For a medium represented by a transfer matrix $\boldsymbol{T}$, reflection of a right-going wave from the leftmost interface is given by $r_{R}=-T_{21} / T_{22}$, while reflection of a left-going wave from the rightmost interface is given by $r_{L}=T_{12} / T_{22}$ (see, e.g., $[17,20])$.

[23] S. M. Barnett, B. Huttner, R. Loudon, and R. Matloob, J. Phys. B: At., Mol. Opt. Phys. 29, 3763 (1996).

[24] S. Scheel, L. Knöll, and D.-G. Welsch, Phys. Rev. A 60, 4094 (1999).

[25] We also note that the electric and magnetic coefficients are equal due to impedance matching and hence need not be referred to separately.

[26] S. A. Ellingsen, J.Phys. A: Math. Theor. 40, 1951 (2007).

[27] U. Leonhardt and W. M. R. Simpson, Phys. Rev. D 84, 081701 (2011). 